\title{
Role of Contrast Enhanced Computed Tomography in Evaluation of Sonographically Detected Focal Lesions in Liver
}

\author{
Sanjay $P^{1}$, Vinod ${ }^{2}$ \\ ${ }^{1}$ Assistant Professor, Department of Radiodiagnosis, Mysore Medical College and Research Institute, Mysore, Karnataka, \\ ${ }^{2}$ Assistant Professor, Department of Radiodiagnosis, Navodaya Medical College Hospital and Research Center, Raichur, \\ Karnataka, India
}

Corresponding author: Dr. Vinod, Assistant Professor, Department of Radiodiagnosis, Navodaya Medical College Hospital and Research Center, Raichur, Karnataka, India

DOI: http://dx.doi.org/10.21276/ijcmsr.2020.5.1.47

How to cite this article: Sanjay P, Vinod. Role of contrast enhanced computed tomography in evaluation of sonographically detected focal lesions in liver. International Journal of Contemporary Medicine Surgery and Radiology. 2020;5(1):A219-A223.

\section{A B S T R A C T}

Introduction: Focal liver lesions are any benign or malignant lesion of variable size in the liver other than the normal parenchyma with or without causing structural and functional abnormality of the hepatobiliary system.

Aim: To evaluate the role of triphasic computed tomography (CT) in detection and characterization of focal liver lesions and help in deciding further course of management

Material and Methods: In this prospective study, 50 patients with sonographically detected focal hepatic lesions were recruited and their triphasic multidetector computed tomography (MDCT) scan findings were evaluated. SPSS version 22 and electronic Microsoft Excel spreadsheets were used for data analysis. Categorical data has been represented as frequency (number) and proportions (percentages), continuous data as Mean \pm S.D. ANOVA and Chi-square tests were used. The confidence level was kept at $95 \%$, hence a $p$-value $<0.05$ was considered as statistically significant.

Results: In our study, the majority (i.e. 40\%) of the patients with metastases were in the age range of 50-59 years with male preponderance (56\%) as compared to females that accounted for (44\%) of the cases. In total 195 focal liver lesions were seen in 50 patients and there were 113 hypovascular and 82 hypervascular lesions. Most hypovascular lesions were best detected during the portal venous phase (PVP) and most hypervascular lesions were in the hepatic-arterial phase (HAP). A significant difference in conspicuity was identified between the HAP and other phases when the lesion size was less than $3 \mathrm{~cm}(p<0.001)$. No patient in our study had a lesion that was detected on unenhanced phase images that were not identified on HAP or PVP images. $100 \%$ of hypovascular lesions with hypo/hypo(cyst)/hypo enhancement patterns were confirmed as cysts. When an enhancing rim in the arterial phase was observed with hyper (rim)/hypo/hypo pattern, all the lesions (27 of 27) were metastases. Hypo/hypo/hyper enhancing pattern, was seen in $6(100 \%)$ lesions, were metastases. Similarly, $100 \%$ hypervascular lesions $A$ (puddles)/A/A enhancement pattern were hemangiomas. 16 of 22 hepatocellular carcinomas (HCC) presented as A (variegated)/A/A(delayed), enhancing pattern. With mixed/mixed/mixed enhancement pattern, all the lesions (21 of 21 ) were metastases. Most (58.8\%) of the malignant hyper/A/A lesions were metastases and the rest of the lesions (35\%) were HCC. The benign lesions were adenoma (5.1\%).

Conclusion: Triphasic CT of the liver is a standardized CT procedure, enables in detection and characterization of the vast majority of focal liver lesions in the presence of different pathological conditions and multilevel disease.

Keywords: Triphasic CT, Focal lesions, Hypovascular, Hypervascular, HAP, PVP

\section{INTRODUCTION}

Focal liver lesions can be defined as any lesion in the liver other than the normal parenchyma with or without causing structural and functional abnormality of the hepatobiliary system and can be of variable size. These lesions can be benign or malignant. The prevalence of various liver lesions has marked differences across geographic regions and ethnic groups. ${ }^{1}$ In a patient without known cancer or history of chronic liver disease, these lesions usually can be evaluated with serial follow-up imaging tests because nearly all are be benign. In patients with cancer, however, prompt determination of the cause of such lesions may be pivotal for defining prognosis and therapy. Small hepatic lesions are deemed benign in $51 \%$ of the $82 \%$ of patients with a known underlying malignancy. ${ }^{2}$ Benign hepatic tumors have been reported in up to $52 \%$ of the general population. ${ }^{3}$ It is therefore important to differentiate between benign and malignant focal liver lesions for further management of the patient.

With the introduction of microbubble contrast agents, contrast-enhanced ultrasonography (CEUS) offers the unique capability to perform continuous, real-time assessment of normal hepatic parenchyma, hepatic vessels, 
and liver lesions. ${ }^{4}$ This extends the temporal window of a dynamic imaging study beyond the limits of standard contrast-enhanced computed tomography (CT) and magnetic resonance imaging examinations, which are able to display static views only. CT is the imaging modality most often used to evaluate focal liver lesions; however, the complex blood supply of the liver frustrates the search for an optimal contrast-enhanced CT protocol for the detection and characterization of focal hepatic lesions. Although the liver receives approximately $30 \%$ of its blood supply from the hepatic artery and $70 \%$ from the portal vein - most primary and secondary liver neoplasms receive $80-95 \%$ of their blood supply from the hepatic artery. Because of a high frequency of benign focal liver lesions such as cysts, haemangiomas, and focal nodular hyperplasia, characterization of these lesions is essential. Consequently, the preferred liver CT technique should combine a high sensitivity for lesion detection with a good ability for lesion characterization, to differentiate lesions that do need further diagnostic tests or treatment for lesions that do not. To meet these requirements, a triphasic spiral CT technique was developed to image the entire liver in arterial, portal, and equilibrium phases. ${ }^{5,6}$ Although the current literature search shows that magnetic resonance imaging (MRI) has a comparable rate in detection and classification of focal liver lesions, however, rapid availability and short scanning time made CT an ideal imaging technique. ${ }^{7-9}$ With these viewpoints, the present was conducted to assess the role of triphasic computed tomography in the detection and characterization of focal liver lesions and help in deciding further course of management.

\section{MATERIAL AND METHODS}

This prospective, observational study was conducted in the radiodiagnosis department of a tertiary care teaching hospital over a period of one and a half years on 50 patients of all age groups with sonographically detected focal hepatic lesions. Informed written consent was taken from patients about participation in the present study. Ethical clearance was sought and approved by the institutional ethical committee before the conduct of the study.

\section{Study Protocol}

A total of 50 patients of all age groups of both sexes with ultrasound proven cases of hepatic lesions was included for the study. The patients with suspected liver pathologies were subjected to detailed ultrasound machines. In the second step, patients were evaluated with Triphasic CT using 16 slice-MDCT scanners.

\section{Triphasic CT Imaging Technique of Liver}

Patients were kept nil orally 4 hours prior to the CT scan to avoid complications while administrating contrast medium. Routine anteroposterior topogram of the abdomen was initially taken in all patients in the supine position with the breath held. Axial sections of $5 \mathrm{~mm}$ thickness were taken from the level of lung bases to the level of ischial tuberosities. In all cases, a plain scan was followed by an intravenous contrast scan in suspended inspiration. For contrast enhancement, 18G Vasofix (indwelling catheter) was placed in the antecubital vein and dynamic injection at a rate of about $80-100 \mathrm{cc}$ of non-ionic contrast material (ultravist: iopromide; $300 \mathrm{mg}$ iodine/ml) was given using a power injector. Sections were taken in HAP (40s), PVP (60s) and delayed phases (3-5 mins) in the craniocaudal direction from the superior margin to the inferior border of the liver. Post-study reconstructions were done at $2.5 \mathrm{~mm}$. Sagittal and coronal reconstructions were made wherever necessary. Newer techniques in multislice CT like curved planar reformatting, volume rendering, maximum and minimum intensity projections were done as and when necessary.

\section{Image Interpretation}

Viewing of all reconstructed images was done. First, the unenhanced, HAP and PVP images were reviewed for the presence of focal liver lesions. Second, the CT appearance of each lesion in each phase (unenhanced, HAP, PVP and delayed images) were characterized based on the enhancement patterns and its attenuation compared with that of the liver parenchyma in that phase. Lesions were broadly grouped as hypervascular or hypovascular lesions relative to the surrounding parenchyma. Images of different phases were analyzed separately and later were reinterpreted together. Later the nature of lesions confirmed by biopsy/ surgery / USG / follow-up as and when required. In some patients with multiple lesions, a biopsy was performed on only one or two lesions. Rest with similar CT appearance was assumed to be the same lesion. If the lesion did not show any change in size after a minimum of six months then the lesion was presumed to be benign. If the number of lesions was more than 10 then the analysis of 10 most representative lesions was performed using the combination of all the phases. The appearance of each lesion in each phase was described on the basis of attenuation and homogeneity of the lesion in comparison to the liver parenchyma in that phase and was expressed as one of the five possible states:

1. Area of water attenuation, homogenous: hypo(cyst)

2. Area of soft-tissue attenuation, slightly in homogenous: hypo

3. Area of mixed attenuation but hypoattenuating than the arterial system: mixed

4. Area of hyper attenuation but less than the arterial system: hyper

5. Isoattenuation compared to the arterial system: arterial or A

6. The pattern of enhancement is a three-pattern name that includes the appearance of the lesion in each phase (e.g.; hypo/hypo/hypo)

7. Additional patterns of subtype enhancement in arterial phases like peripheral puddles, variegated, continuous hyperattenuating rim, incomplete rim, and cleft were also considered.

\section{STATISTICAL ANALYSIS}

The data were entered in Microsoft Excel sheet and were analyzed using SPSS (Statistical Package for Social Sciences) version 22 software. The categorical data was represented in the form of frequency and percentage. ANOVA/Chi-square tests were used to test the significance of qualitative data. Continuous data were represented as mean and standard deviation. $\mathrm{p}$-value $<0.05$ was considered as significant. 


\section{RESULTS}

In our study, a majority (i.e. 40\%) of the patients with metastases were in the age range of 50-59 years with male preponderance $(56 \%)$ as compared to females who accounted for $(44 \%)$ of the cases. [Table 1]

With regard to the detection of lesions, in the present study, a total of 195 focal liver lesions were seen in 50 patients and there were 113 hypovascular and 82 hypervascular lesions. Most hypovascular lesions were best detected during PVP and most hypervascular lesions were in HAP. [Table-2]

\begin{tabular}{|l|c|c|c|}
\hline SI. No. & Characteristics & No. of Cases & Percentage \\
\hline \multirow{4}{*}{1.} & Age (in years) & & \\
\cline { 2 - 4 } & $<30$ & 02 & 04.00 \\
\cline { 2 - 4 } & $30-39$ & 08 & 16.00 \\
\cline { 2 - 4 } & $40-49$ & 12 & 24.00 \\
\cline { 2 - 4 } & $50-59$ & 17 & 34.00 \\
\cline { 2 - 4 } & $60-69$ & 08 & 16.00 \\
\cline { 2 - 4 } & $70-79$ & 03 & 06.00 \\
\hline 2. & Gender & & \\
\cline { 2 - 4 } & Male & 28 & 56.00 \\
\cline { 2 - 4 } & Female & 22 & 44.00 \\
\hline
\end{tabular}

Table-1: Demographic profile of cases enrolled in the study $(\mathrm{N}=50)$

\begin{tabular}{|l|l|c|c|}
\hline Sl. No. & Characteristics & $\begin{array}{c}\text { No. of } \\
\text { Lesions }\end{array}$ & Percentage \\
\hline \multirow{3}{*}{1.} & Group & & \\
\cline { 2 - 4 } & Benign & 57 & 29.23 \\
\cline { 2 - 4 } & Malignant & 138 & 70.77 \\
\hline \multirow{2}{*}{2.} & Group & & \\
\cline { 2 - 4 } & Hypovascular lesions & 113 & 57.95 \\
\cline { 2 - 4 } & Hypervascular lesions & 82 & 42.05 \\
\hline
\end{tabular}

Table-2: Distribution of focal lesions of the liver among study subjects
In our study, a greater number of hypovascular lesions were identified with greater lesion conspicuity on the PVP than on other phases especially when the size of the lesion was less than $3 \mathrm{~cm}$. When the lesions were $>3 \mathrm{~cm}$ no statistically significant difference was seen between PVP and HAP. In our study, the number of hypervascular lesions seen was higher on HAP than on PVP and unenhanced phase when the lesion size was less than $3 \mathrm{~cm}$ in size. A significant difference in conspicuity was identified between the HAP and other phases when the lesion size was less than $3 \mathrm{~cm}$ but no significant difference when the lesion size was greater than $3 \mathrm{~cm}$ in size. In our study, the unenhanced scan had lower sensitivity for the identification of small lesions because of difficulty in differentiating it from unenhanced vessels and biliary dilatation. Most of the differences were seen when the lesion size was less than $3 \mathrm{~cm}$ because larger lesions were seen in all phases. No patient in our study had a lesion that was detected on unenhanced phase images that were not identified on HAP or PVP images. [Figure 3]

In the characterization of hypovascular lesions, the first distinction was made between cysts and hypovascular solid lesions. All the 21 (100\%) lesions with hypo/hypo(cyst)/hypo enhancement patterns were confirmed to be cysts because of sharp margins and homogenous hypovascular pattern. When

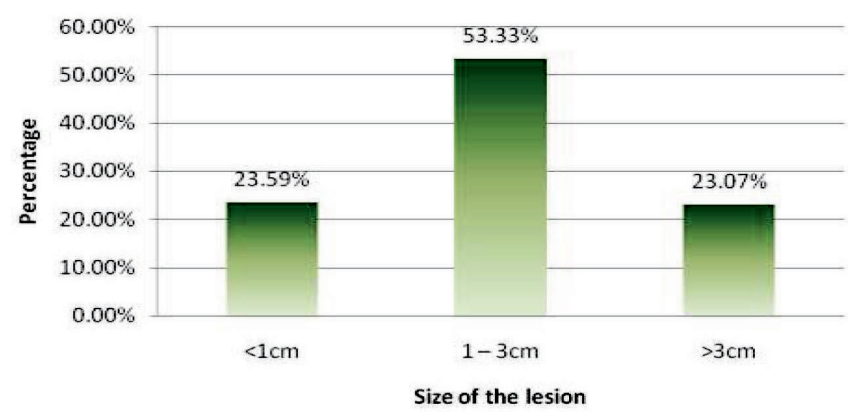

Figure-3: Size-wise distribution pattern of focal lesions of the liver

\begin{tabular}{|c|c|c|c|c|c|c|c|c|c|}
\hline \multicolumn{3}{|l|}{ Enhance Pattern } & \multirow{2}{*}{$\begin{array}{c}\text { Total } \\
\text { No. of } \\
\text { Lesions }\end{array}$} & \multicolumn{3}{|c|}{ Malignant Lesions } & \multicolumn{3}{|c|}{ Benign Lesions } \\
\hline HAP & PVP & DP & & No. & $\%$ & $\begin{array}{l}\text { Final } \\
\text { Diagnosis }\end{array}$ & No. & $\%$ & $\begin{array}{l}\text { Final } \\
\text { Diagnosis }\end{array}$ \\
\hline \multicolumn{10}{|c|}{ Hypovascular Enhancing Patterns } \\
\hline Hypo & Hypo(cyst) & Hypo & $\mathrm{n}=21$ & 00 & NA & NA & 21 & 100 & Cyst \\
\hline hyper & Hypo(cyst) & Hypo & $\mathrm{n}=8$ & 00 & NA & NA & 08 & 100 & Abscess \\
\hline Hyро & Hypo & Hypo & $n=51$ & 51 & 100 & Metastases & NA & NA & NA \\
\hline hyper & Hypo & Нyро & $\mathrm{n}=27$ & 27 & 100 & Metastases & NA & NA & NA \\
\hline Hypo & Hypo & hyper & $\mathrm{n}=06$ & 06 & 100 & Metastases & NA & NA & NA \\
\hline \multicolumn{10}{|c|}{ Hypervascular Enhancing Patterns } \\
\hline A (Puddles) & A & A & $\mathrm{n}=26$ & 00 & 00 & NA & 26 & 100 & Hemangiomas \\
\hline A & A & $\mathrm{A}$ (Cleft) & $\mathrm{n}=01$ & 00 & 00 & NA & 01 & 100 & FNH \\
\hline A (Variegated) & A & A (Capsule) & $\mathrm{n}=16$ & 16 & 100 & $\mathrm{HCC}$ & & & \\
\hline Hyper (Incomplete) & A & A & $\mathrm{n}=01$ & 01 & 100 & $\begin{array}{l}\text { Intrahepatic } \\
\text { CCA }\end{array}$ & & & \\
\hline Mixed & Mixed & Mixed & $\mathrm{n}=21$ & 21 & 100 & Metastases & & & \\
\hline \multirow[t]{2}{*}{ Hyper } & A & A & $\mathrm{n}=17$ & 06 & 35.30 & $\mathrm{HCC}$ & 01 & 5.9 & Adenoma \\
\hline & & & & 10 & 58.8 & Metastases & & & \\
\hline \multicolumn{10}{|c|}{$\mathrm{HAP}=$ hepatic arterial phase; $\mathrm{PVP}=$ portal venous phase; $\mathrm{DP}=$ delayed phase } \\
\hline
\end{tabular}




\begin{tabular}{|l|c|c|c|c|c|c|}
\hline Diagnosis & Sensitivity & Specificity & PPV & NPV & Accuracy & p-value \\
\hline Abscesses & 100.00 & 100.00 & 100.00 & 100.00 & 100.00 & $<0.001$ \\
\hline Adenoma & 00.00 & 99.98 & 00.00 & 99.98 & 99.98 & - \\
\hline Simple cyst & 100.00 & 100.00 & 100.00 & 100.00 & 100.00 & $<0.001$ \\
\hline Hemangioma & 100.00 & 100.00 & 100.00 & 100.00 & 100.00 & $<0.001$ \\
\hline FNH & 100.00 & 100.00 & 100.00 & 100.00 & 100.00 & $<0.005$ \\
\hline HCC & 81.81 & 100.00 & 100.00 & 97.74 & 97.74 & $<0.001$ \\
\hline Intrahepatic CCA & 100.00 & 100.00 & 100.00 & 100.00 & 100.00 & $<0.005$ \\
\hline Metastases & 93.91 & 100.00 & 100.00 & 91.95 & 96.41 & $<0.001$ \\
\hline \multicolumn{2}{|l|}{ Table-5: Correlation of CT enhancement pattern in the diagnosis of focal liver lesions with a final diagnosis } \\
\hline
\end{tabular}

enhancing rim was observed, with hyper (rim)/hypo(cyst)/ hypo enhancing pattern, all the lesions were abscess. In noncystic hypovascular lesions, when the lesions demonstrated hypo/hypo/hypo enhancing pattern, 51(100\%) lesions were metastases. When an enhancing rim in the arterial phase was observed with hyper (rim)/hypo/hypo pattern, all the lesions (27 of 27) were metastases. Hypo/hypo/hyper enhancing pattern, was seen in 6(100\%) lesions, were metastases. Similarly, in the characterization of hypervascular lesions: all the 26 (100\%) lesions with A (puddles)/A/A enhancement pattern were hemangiomas. A/A/A (cleft) enhancement pattern, was seen in 1 case of FNH and hyper (incomplete)/ $\mathrm{A} / \mathrm{A}$ enhancement pattern, which were seen in 1 case of Intrahepatic CCA. 16 of 22 hepatocellular carcinomas (HCC) presented as A (variegated)/A/A(delayed), enhancing pattern. With mixed/mixed/mixed enhancement pattern, all the lesions (21 of 21) were metastases. Most (58.8\%) of the malignant hyper/A/A lesions were metastases and the rest of the lesions $(35 \%)$ were HCC. The benign lesions were Adenoma (5.1\%). Interpretation of hyper/A/A enhancing pattern should be done in a clinical context, a biopsy is essential for differentiating these lesions. [Table-4; Table-5].

\section{DISCUSSION}

In the detection of lesions, our findings were similar to the study done by Miller F et al., on a suspected or known case of malignant focal lesions. ${ }^{10}$ In our study, we grouped lesion size as $<1 \mathrm{~cm}, 1-3 \mathrm{~cm}$, and $>3 \mathrm{~cm}$. Miller $\mathrm{F}$ et al., in their study grouped the lesion size as $<1 \mathrm{~cm}, 1-2 \mathrm{~cm}, 2-3 \mathrm{~cm}$, and $>3 \mathrm{~cm}$. They found that in patients with hypovascular malignancies, a significant difference was seen in the number of lesions detected between PVP and other phases when the lesion was $<2 \mathrm{~cm}$. They also detected that the conspicuity of the hypovascular lesions was higher on the PVP than on other phases when the lesions were $<3 \mathrm{~cm}$. In patients with hypervascular malignancies, a larger number of lesions in their study were seen on the HAP than in other phases when the lesions were $<2 \mathrm{~cm}$. The conspicuity of lesions was higher on HAP and a significant statistical difference was seen between the PVP and HAP, between PVP and unenhanced phases, and between HAP and unenhanced phases when the size of the lesion was $<3 \mathrm{~cm} \cdot{ }^{10}$ Francis IR et al., reported that patients with suspected or known hepatic tumors demonstrated that maximal tumor-to-parenchyma differences are achieved on PVP images. ${ }^{6}$ In another study by Soyer $\mathrm{P}$ et al., on the detection of hypovascular hepatic metastases at triphasic liver $\mathrm{CT}$ reported that the PVP images depicted significantly more hypovascular metastases than in any other phases. ${ }^{11}$ Miller $\mathrm{F}$ et al., reported that no lesions were detected on an unenhanced phase that was not seen on other phases, hypervascular lesions were best detected on HAP, and hypovascular lesions were more detected on PVP. However not all the hypervascular lesions detected on HAP were malignant suggesting that benign lesions may also be hypervascular [focal nodular hyperplasia (FNH), Hepatic adenoma, Peliosis Hepatis]. ${ }^{10}$

In characterization of lesions, the present study was similar and correlated well with the study done by van Leuwen MS et al. Wherein, they found 11 patterns of enhancement and demonstrated that six of the 11 enhancement patterns were always due to benign disease, three of the 11 patterns were always due to malignant disease and the other two patterns was due to metastases and hemangiomas. ${ }^{12}$

In the present study, we had 11 patterns of enhancement. Four of the 11 enhancement patterns were always due to benign lesions, another five of 11 enhancement patterns were always due to malignant lesions, and the other two of the 11 enhancement patterns were due to both malignant and benign lesions. We found two different enhancement patterns one for the abscess and another for intrahepatic clear cell adenocarcinoma (CCA). These findings were contradictory to the study conducted by van Leuwen MS et al. ${ }^{12}$

The present study also correlated well with the study conducted by Gualdi GF et al., in their study to evaluate the role of triphasic $\mathrm{CT}$ in the characterization of non-cystic focal lesions on sixty- six they found, 11 patterns of enhancement of the lesions in different phases. Four of 11 enhancement patterns (hypo/hyper/hyper, hyper/iso/iso, hyper/hyper/iso, and hyper/hyper/hyper) were always referable to benign disease. Four of 11 enhancement patterns (iso/hypo/hypo, iso/iso/hypo, hyper/hypo/hypo, and hyper/hyper/hypo) were always referable to malignant disease (hepatocellular carcinoma-HCC metastases). The other three patterns (hypo/hypo/hypo, hypo/hypo/hyper, and hyper/A/A) were seen in both benign and malignant diseases. ${ }^{13}$

The present study was also comparable to the study conducted by Nino-Murcia $\mathrm{M}$ et al. wherein, enhancement patterns of a dominant lesion (in multiple lesions) or a represented lesion in each patient classified into one of the five categories of enhancement: homogenous, abnormal internal vessels or variegated, peripheral puddles, complete ring or incomplete ring. Lesions without enhancement were recorded separately. Histologic examination, correlative imaging, or clinical or imaging follow up were used as the standards of reference. 
It was observed that $92 \%$ of the 100 lesions demonstrated arterial phase enhancement. The patterns associated with PPV of $82 \%$ or more and specificity of $80 \%$ or more included abnormal internal vessels or variegated (HCC), peripheral puddles (Hemangiomas), and complete ring (Metastases). ${ }^{14}$

\section{CONCLUSION}

In conclusion, Triphasic CT of the liver is a standardized CT procedure, enables in detection and characterization of the vast majority of focal liver lesions in the presence of different pathological conditions and multilevel disease.

\section{Limitation}

The limitation of the present study is broader inclusion criteria and limited sample size. Owing further studies on the larger size with objective criteria of inclusion are recommended in order to get a decisive account of focal lesions of the liver.

\section{REFERENCES}

1. Méndez-Sánchez N, Villa AR, Chávez-Tapia NC, Ponciano-Rodriguez G, Almeda-Valdés P, González $\mathrm{D}$, et al. Trends in liver disease prevalence in Mexico from 2005 to 2050 through mortality data. Annals of Hepatology 2005;4(2):52-55.

2. Schwartz LH, Gandras EJ, Colangelo SM, Ercolani MC, Panicek DM. Prevalence and importance of small hepatic Lesions Found at CT in Patients with cancer. Radiology 1999;210(1):71-74.

3. Karhunen PJ. Benign hepatic tumours and tumour-like conditions in men. J Clin Pathol 1986;39(4):183-188.

4. Brannigan M, Burns PN, Wilson SR. Blood flow patterns in focal liver lesions at microbubble-enhanced US. Radiographics 2004;24(5):921-935.

5. Bonaldi VM, Bret PM, Reinhold C, Atri M. Helical computed tomogram of liver, value of an early hepatic arterial phase Radiology 1995;197(2):357-63.

6. Francis IR, Cohan RH, McNulty NJ, Platt JF, Korobkin M, Gebremariam A, et al. Multidetector CT of the liver and hepatic neoplasms: Effect of multiphasic imaging on tumor conspicuity and vascular enhancement. AJR Am J Roentgenol 2003;180(5):1217-1224.

7. Ichikawa T, Saito K, Yoshioka N, Tanimoto A, Gokan T, Takehara $Y$ et al. Detection and characterization of focal liver lesions: a Japanese phase III, multicenter comparison between gadoxetic acid disodium enhanced magnetic resonance imaging and contrast enhanced computed tomography predominantly in patients with hepatocellular carcinoma and chronic liver disease. Invest Radiol 2010;45(1):133-41.

8. Hammerstingl R, Huppertz A, Breuer J, Balzer T, Blakeborough A, Carter R et al. Diagnostic efficacy of gadoxetic acid (Primovist)-enhanced MRI and spiral CT for a therapeutic strategy: comparison with Intraoperative and histopathologic findings in focal liver lesions. Eur Radiol 2008;18(6):457-467.

9. Soyer P, Sirol M, Fargeaudou Y, Duchat F, Hamzi L, Boudiaf M, et al. Differentiation between true focal liver lesions and psudolesions in patients with fatty liver: evaluation of helical CT criteria. Eur Radiol 2010; 20(5): 1726-37.

10. Miller F, Butler R, Hoff F, Fitzgerald S, Nemcek Jr
AA, Gore R. Using triphasic helical CT to detect focal hepatic lesions in patients with neoplasms. AJR. American journal of roentgenology. 1998;171(3):643-9.

11. Soyer P, Poccard M, Boudiaf M, Abitbol M, Hamzi L, Panis Y, Valleur P, Rymer R. Detection of hypovascular hepatic metastases at triple-phase helical CT: sensitivity of phases and comparison with surgical and histopathologic findings. Radiology. 2004;231(2):41320.

12. van Leeuwen MS, Noordzij J, Feldberg MA, Hennipman AH, Doornewaard H. Focal liver lesions: characterization with triphasic spiral CT. Radiology. 1996;201(2):327-36.

13. Gualdi GF, Casciani E, D'Agostino A, Polettini E. Triphasic spiral computerized tomography of the liver: vascular models of non-cystic focal lesions. La Radiologia medica.1998;96(4):344-52.

14. Nino-Murcia M, Olcott EW, Jeffrey Jr RB, Lamm RL, Beaulieu CF, Jain KA. Focal liver lesions: pattern-based classification scheme for enhancement at arterial phase CT. Radiology. 2000;215(3):746-751.

\section{Source of Support: Nil; Conflict of Interest: None}

Submitted: 14-02-2020; Accepted: 27-02-2020; Published online: 20-03-2020 